\title{
Peaceman Rachford Alternating Direct Implicitly Method for Linear Advection-Diffusion Equation and Its Application
}

\author{
Kedir Aliyi Koroche \\ Department of Mathematics, College of Natural and Computational Sciences, Ambo University, Ambo, Ethiopia \\ Email address: \\ kediraliyi39@gmail.com \\ To cite this article: \\ Kedir Aliyi Koroche. Peaceman Rachford Alternating Direct Implicitly Method for Linear Advection-Diffusion Equation and Its Application. \\ International Journal of Discrete Mathematics. Vol. 6, No. 1, 2021, pp. 5-14. doi: 10.11648/j.dmath.20210601.12
}

Received: April 3, 2021; Accepted: May 6, 2021; Published: May 14, 2021

\begin{abstract}
In this paper, Peaceman-Rachford alternating direct implicitly methods presented and applied for solve linear advection-diffusion equation. First, the domain was discretized using the uniform mesh of step length and time step. Secondly, by applying the Taylor series methods, we discretize partial derivative of governing equation and we obtain the central difference equation for Partial differential equation of given governing equation in both duration. Then rearranging the obtained central difference equation; we write the two half scheme of the present method. From each half of these schemes, we obtain tri-diagonal coefficient matrices associated with the system of difference equation. Lastly by applying the Thomas algorithm and writing MATLAB code for the scheme we obtain solution of the governing linear advection diffusion equation. To validate the applicability of the proposed method, three model examples are considered and solved for different values of mesh sizes in both directions. The convergence has been shown in the sense of maximum absolute error $\left(\mathrm{L}_{1}\right.$-norm) and $\mathrm{L}_{2}$ norm, numerical error and experimental order of convergence. The stability and convergence of the present numerical method are also guaranteed and the comparability of numerical solution and the stability of the present method are presented by using the graphical and tabular form. The numerical results presented in tables and graphs confirm that the approximate solution is in good agreement with the exact solution.
\end{abstract}

Keywords: Linear Advection-diffusion Equation, Peaceman-Rachford Alternating Direct Implicitly Method, Taylor Series Methods, Tri-diagonal Coefficient Matrices, Thomas Algorithm, Stability and Convergence

\section{Introduction}

Advection-diffusion equation is a parabolic partial differential equation which is derived on the principle of conservation of mass using Fick's $1^{\text {st }}$ law [11]. Particles, energy, or physical quantities are transferred inside a physical system [11] due to two processes: advection and diffusion [11]. The advection-diffusion equation describes the set of processes for several substances [11].

The significant applications of the advection-diffusion equation lie in fluid dynamics, heat transfer, and mass transfer [1]. The diffusion-convection-reaction equation arises in several physical phenomena, such as the dispersion of chemicals in reactors [2], the shocks in hydrodynamical flows, the mass transfer in the capillary membrane [2] bioreactor, and the tracer dispersion in the porous medium. Mathematically, the class of equations can be expressed as a set of partial differential equations (PDEs) with the initial or boundary conditions [2]. Most physical quantities are involved with the interaction of the temporal and spatial domain [2]. This equation is used in the momentum conservation part of the Navier-Stokes equations [3]. The non linear advection-diffusion type equation is one of the popular and important models describing many phenomena derived from various areas of mathematical physics and engineering fields such as gas dynamics, hydrodynamics, shockwaves, heat conduction, and so on [4]. Also, the type of equation represents the Burgers equation, the heat conduction equation, the non linear Schrödinger equation, the Navier-Stokes equation [4]. Hence, the development of efficient and accurate algorithms for solving the equations is of great importance in the computational fluid dynamics community and has been widely studied by many researchers [4].

Advection-diffusion problems arise when the time evolution of chemical or biological species in a flowing medium is described [15]. The blending of particulate 
materials is a common manufacturing unit operation in many industries, such as those that produce chemicals, pharmaceuticals, food products, and agrochemicals [5]. For instance, multi-scale modeling approach for predicting the blending of particulate material in a rotating cylindrical drum is an advection-diffusion equation [5]. Solute dispersion under the combined effects of diffusion and advection in the hetero generous porous medium is modeled by parabolic advection-diffusion equations (ADE). Many harmful effects on humans and the environment such us atmospheric pollutions, contaminated flows in ground water aquifers, chemicals and migration of contamination in the sea waters and river systems, tracer dispersion in a porous medium are modeled by such types of equations $[6,11]$. It also considers physical phenomena where in the diffusion process particles are moving with a certain velocity from higher concentration to lower concentration [11]. The analytical and numerical solutions along with an initial condition and two boundary conditions aid to understand the pollutant concentration distribution behavior through an open medium like air, rivers, lakes, and porous medium. River water pollution can be established by the one-dimensional advection-diffusion equation [11]. It has wide applications in other disciplines too, like soil physics, petroleum engineering, chemical engineering, and biosciences [11]. The practitioners of computational fluid dynamics have decomposed the analysis of the complexity and stiffness of the Navier-Stokes equations in to simpler problems like the Stokes (linear) equations that embody the difficulties of the space discretization of the velocity and pressure fields and the advection-diffusion problem that is related to the transport character of the non-linear terms [7]. This last class of problems includes the non-linear Burgers equations and the linear advection-diffusion equation. In this paper, we will address the one-dimensional linear advection-diffusion equation with homogeneous Dirichlet boundary conditions as this is a meaningful test for established of discrete schemes. For high Reynolds number flows the advection is dominating diffusion but the presence of the boundaries imposing no-slip wall conditions complicates the solution of the problem. Boundary layers develop and in most cases influence deeply the flow dynamics. No-slip wall boundary condition impedes the general use of periodic Fourier representation and spectral calculation [7]. Even though the linear advectiondiffusion equation is difficult to find a closed-form analytical solution in the literature. Most of the efforts have been devoted to the solution of linear advection-diffusion equation with an upstream boundary condition and a Robin or Neumann downstream condition. The presence of the gradient condition at the exit of the domain eases the development of the analytical solution. In their study, Pérez Guérrero et. al. [10] uses a change of variable to obtain a heat equation and then solved it by using a generalized integral transform technique which proposed by Cotta [8]. In their study, van Genuchten et al. [9] can use a variable transformation that reduces the partial differential equation to an ordinary differential equation and they obtain solution of governing partial differential equation which is expressed by the complementary error function [7].

Moreover, in the literature review, there are different numerical methods applied by the author to solve linear advection-diffusion equations [16]. However, still, the accuracy and stability of the method need attention because the treatment of the method used to solve the linear advection-diffusion equation is not trivial distribution. Even though the accuracy and stability of the aforementioned methods need attention, may they require large memory and /or long computational time. So the treatments of this method present ever difficulties that have to be addressed to ensure the accuracy and stability of the solution. To this end, this paper aims to develop and present the accurate and stable numerical method which is Peaceman-Rachford alternating direct implicitly scheme that is capable of solving linear advection-diffusion equation and approximate the exact solution. The convergence has been shown in the sense of maximum absolute error $L_{1}$-norm and $L_{2}$-norm, numerical error $E_{\text {num }}$ and so that the local behavior of the solution is captured exactly. The stability and convergences of the present methods are also investigated.

The present paper is organized as follows. Section two is Statement of the problem, section three is Formulation of the numerical scheme, section four is Stability and convergence analysis for the approximate solution, section five is Numerical results, and section six is Discussion and conclusion.

\section{Statement of the Problem}

Consider that the following linear Advection diffusion equation:

$$
u_{t}+a u_{x}=d u_{x x}+f(x, t),(x, t) \in(a, b) \times(0, T)
$$

with initial and boundary condition respectively

$$
\begin{gathered}
u(x, 0)=u_{0}(x), a \leq x \leq b \\
u(a, t)=0=u(b, t), 0 \leq t \leq T
\end{gathered}
$$

where a and $\mathrm{d}$ are none zero constants and $u_{0}(x)$ and $f(x, t)$ are smooth function on $[a, b] \times[0, T]$. Now we define a mesh size $\mathrm{h}$ and $\mathrm{k}$ and the constant grid point by drawing equidistance horizontal and vertical line of distance ' $h$ ' and ' $k$ ' respectively in ' $x$ ' and ' $t$ ' direction. These lines are called grid line and the point at which the interacting is known as the mesh point. The mesh point that lies at the end of the domain is called the boundary point. The points that lie inside the region are called interiors points. The goal is to approximate the solution ' $u$ ' at the interior mesh points. Hence we discretized the solution domain as:

$$
\begin{gathered}
a=x_{0}<x_{1}<x_{2}<\cdots<x_{M}=b \\
0=t_{0}<t_{1}<t_{2}<\cdots<t_{N}=T
\end{gathered}
$$

Where $x_{j+1}=x_{j}+j h$ and $t_{n+1}=t_{n}+n k,=0(1) M, n=$ $0(1) N$. $\mathrm{M}$ and $\mathrm{N}$ are the maximum numbers of grid points respectively in the $\mathrm{x}$ and $\mathrm{t}$ direction. 


\section{Formulation of the Numerical Scheme}

\subsection{Central Difference Method}

Assuming that $u(x, t)$ has continuous higher order partial derivative on the region $[\mathrm{a}, \mathrm{b}] \mathrm{x}[0, \mathrm{~T}]$. For the sake of simplicity, we use $u\left(x_{j}, t_{n}\right)=u_{j n}, \frac{\partial^{p} u}{\partial x^{p}}=\partial_{x}^{p} u_{j n}$ and $\frac{\partial^{p} u}{\partial t^{p}}=$ $\partial_{t}^{p} u_{j n}$ for $p \geq 1$ is $p^{t h}$ order derivatives. By using Taylor series expansion, we have,

$$
\begin{aligned}
& u_{j+1 n}=u_{j n}+h \partial_{x} u_{j n}+\frac{h^{2}}{2 !} \partial_{x}^{2} u_{j n}+\frac{h^{3}}{3 !} \partial_{x}^{3} u_{j n}+\cdots \\
& u_{j-1 n}=u_{j n}-h \partial_{x} u_{j n}+\frac{h^{2}}{2 !} \partial_{x}^{2} u_{j n}-\frac{h^{3}}{3 !} \partial_{x}^{3} u_{j n}+\cdots \\
& u_{j n+1}=u_{j n}+k \partial_{t} u_{j n}+\frac{k^{2}}{2 !} \partial_{t}^{2} u_{j n}+\frac{k^{3}}{3 !} \partial_{t}^{3} u_{j n}+\cdots \\
& u_{j n-1}=u_{j n}-k \partial_{t} u_{j n}+\frac{k^{2}}{2 !} \partial_{t}^{2} u_{j n}-\frac{k^{3}}{3 !} \partial_{t}^{3} u_{j n}+\cdots
\end{aligned}
$$

Adding the first equation to the second equation, subtract the second equation from the first equation, and again subtract the fourth equation from the third equation of Eq. (4), respectively we obtain:

$$
\begin{gathered}
\partial_{x}^{2} u_{j n}=\frac{u_{j+1}-2 u_{j n}+u_{j-1} n}{h^{2}}+T_{1}, \\
\partial_{x} u_{x}=\frac{u_{j+1}-u_{j-1}}{2 h}+T_{2}, \\
\partial_{t} u_{j n}=\frac{u_{j n+1}-u_{j n-1}}{2 k}+T_{3}
\end{gathered}
$$

Where $T_{1}=-\frac{h^{3}}{12} \partial_{x}^{4} u_{j n} \quad, \quad T_{2}=-\frac{h^{2}}{6} \partial_{x}^{3} u_{j n} \quad$ and $T_{3}=$ $-\frac{k^{3}}{6} \partial_{t}^{3} u_{j n}$ are respectively their local truncation error terms. Now substituting Eq.(5) into Eq.(1) we obtain the difference equation of the form:

$$
\begin{gathered}
\frac{u_{j n+1}-u_{j n-1}}{2 k}+T_{3}+a\left[\frac{u_{j+1 n}-u_{j-1 n}}{2 h}+T_{2}\right] \\
=d\left[\frac{u_{j+1 n}-2 u_{j n}+u_{j-1 n}}{h^{2}}+T_{1}\right]
\end{gathered}
$$

Multiplying both side of this equation by $2 k$ and simplifying it we obtain:

$$
\begin{gathered}
(\beta-\alpha) u_{j+1 n}-2 \beta u_{j n}+(\beta+\alpha) u_{j-1 n}=u_{j n+1}-u_{j n-1}+ \\
T_{j n}
\end{gathered}
$$

Where $\alpha=\frac{a k}{h}, \beta=\frac{2 d k}{h^{2}}$ and $T_{j n}=\left(\frac{a k h^{2}}{3} \partial_{x}^{3}+\frac{k^{3}}{3} \partial_{t}^{2}+\right.$ $\left.\frac{d k h^{2}}{6} \partial_{x}^{4}\right) u_{j n}$ is local traction error obtained from difference scheme in Eq.(6).

\subsection{Peaceman-Rachford Alternative Direct Implicitly Methods}

Alternative direct implicit methods were first introduced by Peaceman, Douglas, and Rachford for obtaining the solution of parabolic and elliptic Partial differential equations in two or three variables [12]. They develop alternative direct implicitly (ADI) methods to overcome the difficulty to find the solution of PDEs by a different method. The difference equation for PDEs at interior mesh point lead linear system of the equation whose coefficient matrices has at most five none zero entries in each row [13]. For many years splitting methods have proved valuable in the numerical solution of time-dependent, multi (space)-dimensional partial differential equations (PDE's). The general idea of splitting is to attack a multi-dimensional problem in such a way that only onedimensional computations are required. This idea has led to the development of a great variety of so-called alternating direction implicit (ADI) methods, locally one-dimensional (LOD) or fractional step methods, and hopscotch type methods [13]. There for feint his paper is interest to use this idea to solve the advection-diffusion equation.

Now we choose starting point to apply this method. Let $u_{j n}^{0}$ is starting point lies at each interior mesh point of the computational domain $(j, n)$ and our interior-point contains two half. Hence forms Eq. (6) equation of these two half of Peaceman-Rachford ADI methods are:

First half:

$$
(\beta-\alpha) u_{j+1 n}^{p+1 / 2}-2 \beta u_{j n}^{p+1 / 2}+(\beta+\alpha) u_{j-1 n}^{p+1 / 2}=u_{j n+1}^{p}-u_{j n-1}^{p}
$$

Second- half:

$u_{j, n-1}^{p+1}-2 \beta u_{j n}^{p+1}-u_{j n+1}^{p+1}=(\alpha-\beta) u_{j+1 n}^{p+1 / 2}-(\beta+\alpha) u_{j-1 n}^{p+1 / 2}$

Where $\quad p=0,1,2, \ldots, n=1(1) N, j=1(1) M . \quad$ The algorithm in Eq.(7) contains the complete time step of the first half from $n=p$ to $n=p+1 / 2$ and second halffrom $n=p+1 / 2$ to $n=p+1$ in to two sub-step respectively. For $n=p=0$, the initial condition or starting points are preformed for each interior points $(j, n)$. Hence by choosing the starting point $u_{j, n}^{0}$ for each interior point $(j, n)$ we update the scheme for both halves. For each interior point, our starting points are values of the initial condition at each interior point. Note that in the single iteration process we have two half. For instance, in the first iteration process, we have two. So for the first half of the first iteration process, we update the starting point ${ }^{6} u_{j, n}^{0}$ 'row by row of first of Eq. (7). Hence for $n^{\text {th }}$ row we have:

$$
(\beta-\alpha) u_{j+1 n}^{1 / 2}-2 \beta u_{j n}^{1 / 2}+(\beta+\alpha) u_{j-1 n+1}^{1 / 2}=u_{j+1}^{0}-u_{j n-1}^{0}
$$

where $\mathrm{j}=0$ (1) $\mathrm{M}$ and $\mathrm{p}=\mathrm{n}=0$. In this equation, the same value of " $u$ " is known value and they are boundary value that lies at $\mathrm{j}=0$ and $\mathrm{j}=\mathrm{M}$. They are not affected by starting value. The remaining values of ' $u$ ' at the interior point in the scheme are remaining unchanged until the process is finished. Therefore for a fixed value of ' $n$ ' $[16,17]$ in Eq.(8) is applied to all interior mesh points along with the row. Thus the obtained system of the equation contains the $\mathrm{M}$ equation. So these equations contain $N^{t h}$ rows and the system contains NM total equation. The coefficient matrix is tri-diagonal. The solution 
of this iteration expressed in terms of $u_{j+1}^{1 / 2}$. For the second half of the first iteration; we update the value $u_{j+1 n}^{1 / 2}$ that obtained from the first half of the first iteration. Hence the second half of the first iteration generated by the formula

$u_{j n-1}^{1}-2 \beta u_{j n}^{1}-u_{j n+1}^{1}=(\alpha-\beta) u_{j+1, n}^{1 / 2}-(\beta+\alpha) u_{j-1 n}^{1 / 2}$

Where $p=0, n=0$ (1) N. For fixed ' $j$ ', we apply Eq.(9) for all interior- point along the jth column and produced the $\mathrm{N}$ equation. Once again our starting value is the value of $u_{j n}^{1 / 2}$ that obtained first half and the remaining value of " $u$ " at interior point $(j, n)$ remain unchanged. Like the first half, the second half of the first iteration contains the NM equation. The coefficient matrix is a tri-diagonal matrix. The values of solutions for this half stored in terms of $u_{j n}^{1}$. Generally, the successive iteration of the PRADI-method are:

Second iteration (for $\mathrm{p}=1$ ): First half: $(\beta+\alpha) u_{j+1 n}^{3 / 2}-$ $2 \beta u_{j n}^{3 / 2}+(\beta+\alpha) u_{j-1 n}^{3 / 2}=u_{j n+1}^{1}-u_{j, n-1}^{1}, \mathrm{j}=1$ (1) $\mathrm{M}, \mathrm{nth}$ row.

Second half: $u_{j n-1}^{2}-2 \beta u_{j n}^{2}-u_{j n+1}^{2}=(\alpha-\beta) u_{j+1, n}^{3 / 2}-$ $(\beta+\alpha) u_{j-1}^{3 / 2}, \mathrm{n}=1(1) \mathrm{N}, \mathrm{jth}$ column.

Third iteration (for $\mathrm{p}=2$ ):

First half: $(\beta+\alpha) u_{j+1 n}^{5 / 2}-2 \beta u_{j n}^{5 / 2}+(\beta+\alpha) u_{j-1 n}^{5 / 2}=$ $u_{j n+1}^{2}-u_{j, n-1}^{2}, j=1$ (1) M, nth row.

Second half: $u_{j n-1}^{3}-2 \beta u_{j n}^{3}-u_{j n+1}^{3}=(\alpha-\beta) u_{j+1, n}^{5 / 2}-$ $(\beta+\alpha) u_{j-1 n}^{5 / 2}, \mathrm{n}=1(1) \mathrm{N}, \mathrm{jth}$ column.

Fourth iteration (for $\mathrm{p}=3$ )

First half: $(\beta+\alpha) u_{j+1 n}^{7 / 2}-2 \beta u_{j n}^{7 / 2}+(\beta+\alpha) u_{j-1 n}^{7 / 2}=$ $u_{j n+1}^{3}-u_{j, n-1}^{3}, j=1$ (1) M, nth row.

Second half: $u_{j n-1}^{4}-2 \beta u_{j n}^{4}-u_{j n+1}^{4}=(\alpha-\beta) u_{j+1, n}^{7 / 2}-$ $(\beta+\alpha) u_{j-1 n}^{7 / 2}, \mathrm{n}=1(1) \mathrm{N}, \mathrm{jth}$ column.

This process is continuous until the tolerance of the solution is obtained. For all-iteration, we obtain the tri-diagonal matrices and we use the Thomas method to find the solution of each system of iteration. One of the termination conditions is assembling the value of ' $u$ ' at each interior point's into a matrix with the same configuration of the grid point. If the norm of the difference between two successive [18] such matrices is less than a prescribed tolerance, the iteration is terminated.

\subsection{Thomas Method}

The tri-diagonal system of equation arises after in engineering application. It appears in the special form whose coefficients matrix contains three none zero entries with main diagonal. Let us conceder the coefficient matrix of the first iteration given us $A u^{1 / 2}=b^{1 / 2}$ and $B u^{1}=b^{1}$ respectively for both first and second half's where

$$
\begin{gathered}
A=\left[\begin{array}{ccccc}
-2 \beta & \beta-\alpha & 0 & \ldots & 0 \\
\beta+\alpha & 2 \beta & \beta-\alpha & \ldots & 0 \\
0 & \beta+\alpha & \ddots & \vdots & 0 \\
\vdots & \vdots & 0 & \\
0 & 0 & 0 & \beta+\alpha & -2 \beta
\end{array}\right] \\
B=\left[\begin{array}{ccccc}
-2 \beta & 1 & 0 & \ldots & 0 \\
-1 & 2 \beta & 1 & \ldots & 0 \\
0 & -1 & \ddots & \vdots & 0 \\
\vdots & \vdots & & & 0 \\
0 & 0 & 0 & -1 & -2 \beta
\end{array}\right]
\end{gathered}
$$

And the corresponding column vectors are:

$$
\begin{aligned}
b^{1 / 2}= & {\left[\begin{array}{c}
(-\alpha-\beta) u_{j+1,1}^{1 / 2}+u_{j 1}^{0}-u_{1, o}^{0}, \ldots, \\
-(\alpha+\beta) u_{M-11}^{1 / 2}+u_{M-21}^{o}-u_{M 1}^{0}
\end{array}\right]^{t} } \\
b^{1}= & {\left[u_{1 n-1}^{1}+(\alpha-\beta) u_{1 n}^{1 / 2}-u_{1 n+1}^{1 / 2}, \ldots,-u_{1 N}^{1}+\right.} \\
& \left.(\alpha-\beta) u_{2 N-1}^{1 / 2}-(\alpha+\beta) u_{0 N-2}^{1 / 2}\right]^{t}
\end{aligned}
$$

If the prescribed tolerance of iteration is not captured at the first iteration, the solution of this iteration is the starting value for the second iteration. The process is continuous until the tolerance is a capture. To solve these types of system of the equation the most recommended numerical method is the Thomas method. This is because the coefficient matrix contains several zero entries. This method is to use the Gauss elimination technique with the diagonal entries scaled to 1 ine each step. Thus we can write MATLAB code and find the solution to Eq. (1).

\section{Stability and Convergent Analysis}

Since the time step changed from $n=n$ to $n=n+1 / 2$ and $n=n+1 / 2$ to $n=n+1$. Now we want to find the global error bound condition for the scheme. Let $\delta_{j n}$ be the small constant who's their nth sum is less than one for all point ( $j$, n). Hence form Eq. (7) the next succeeding says:

$$
\begin{gathered}
(\beta-\alpha) \tilde{u}_{j+1 n+1}-2 \beta \tilde{u}_{j n}+(\beta+\alpha) \tilde{u}_{j-1 n-1 / 2} \\
=\tilde{u}_{j n}-\tilde{u}_{j n-1}+\gamma \delta_{j n+1 / 2} \\
\tilde{u}_{j n}-2 \beta \tilde{u}_{j-1 n-1}-\tilde{u}_{j n+1}=(\alpha-\beta) \tilde{u}_{j+1 n+1 / 2}- \\
(\beta+\alpha) \tilde{u}_{j-1 n+1 / 2}+\gamma \delta_{j n+1}
\end{gathered}
$$

Where $\gamma>0$ be constant. Now let $u_{j n}$ and $\tilde{u}_{j n}$ two numerical solutions at two were succeeding points. Hence the error between these two numerical solutions is: $\varepsilon_{j n}=\tilde{u}_{j n}-$ $u_{j n}$ for $\mathrm{n}=1$ (1) $\mathrm{N}$ and $\mathrm{j}=0$ (1) $\mathrm{M}$ for $\varepsilon_{j n}$ is a round off error. Subtracting Eq. (7) from Eq. (11), we obtain:

$$
\begin{gathered}
(\beta-\alpha) \varepsilon_{j+1 n+1}-2 \beta \varepsilon_{j n}+(\beta+\alpha) \varepsilon_{j-1 n-1 / 2} \\
=\epsilon_{j n}-\varepsilon_{j n-1}+\gamma \delta_{j n+1 / 2}
\end{gathered}
$$




$$
\begin{gathered}
\varepsilon_{j n}-2 \beta \varepsilon_{j-1 n-1}-\varepsilon_{j n+1}=(\alpha-\beta) \varepsilon_{j+1 n+1 / 2}- \\
(\beta+\alpha) \varepsilon_{j-1 n+1 / 2}+\gamma \delta_{j n+1}
\end{gathered}
$$

Now eliminating the error term $\varepsilon_{j+1 n+1 / 2}$ and $\varepsilon_{j-1 n-1 / 2}$ from Eq.(12) and simplifying it, we obtain:

$$
\begin{gathered}
2 \beta \varepsilon_{j n}+2 \beta \varepsilon_{j-1 n-1}-\varepsilon_{j n+1}=\varepsilon_{j n-1}-\gamma\left(\delta_{j n+1 / 2}+\delta_{j n+1}\right) \\
\widetilde{D} \varepsilon_{j n+1}=I \varepsilon_{j n-1}-\gamma\left(\delta_{j n+1 / 2}+\delta_{j n+1}\right)
\end{gathered}
$$

Where $\breve{D}$ tri-diagonal matrix and I is an identity matrix whose Eigen-value is less than one. From Eq.(13) we obtain

$$
\varepsilon_{j n+1}=\breve{D}^{-1} \varepsilon_{j n-1}-\gamma \breve{D}^{-1}\left(\delta_{j n+1 / 2}+\delta_{j n+1}\right)
$$

Now applying the properties of matrix norm and using triangular in equality on Eq.(14) we obtain:

$$
\begin{gathered}
\left\|\varepsilon_{j n+1}\right\|=\left\|\breve{D}^{-1} \varepsilon_{j n-1}-\gamma \breve{D}^{-1}\left(\delta_{j n+1 / 2}+\delta_{j n+1}\right)\right\| \\
\leq\left\|\breve{D}^{-1} \varepsilon_{j n-1}\right\|+\left\|\gamma \breve{D}^{-1}\left(\delta_{j n+1 / 2}+\delta_{j n+1}\right)\right\| \leq \\
\left\|\breve{D}^{-1}\right\|\left\|\varepsilon_{j n-1}\right\|+\left\|\gamma \breve{D}^{-1}\right\|\left\|\delta_{j n+1 / 2}+\delta_{j n+1}\right\|<\left\|\varepsilon_{j n-1}\right\|+ \\
\gamma G
\end{gathered}
$$

Where $\left\|\breve{D}^{-1}\right\|<1$ and $\left\|\breve{D}^{-1}\right\|\left\|\delta_{j n+1 / 2}+\delta_{j n+1}\right\|<G, \mathrm{G}$ is constant. Let $\mathrm{j}=1$ and $\mathrm{n}=0$. Then from Eq.(15), we obtain $\left\|\varepsilon_{1,1}\right\|<\left\|\varepsilon_{1,-1}\right\|+\gamma G$ impliesthat $\left\|\varepsilon_{1,1}\right\|<\left\|\varepsilon_{0,0}\right\|+$ $\gamma G$. This shows that the global error is bounded. Generally from the above condition, we mean that for any' $(\mathrm{j}, \mathrm{n})$ ' we have a different matrix coefficient of the error term $\widetilde{D}$ and the error is by the norm of this matrix which is given as:

$$
\left\|\varepsilon_{j+1, n+1}\right\|<\left\|\varepsilon_{j n}\right\|+\max _{\substack{1 \leq j \leq M \\ 1 \leq n \leq N}}\left\|\breve{P}_{j n}\right\|
$$

where $\breve{P}_{j n}=\breve{D}^{-1}\left(\delta_{j n+1 / 2}+\delta_{j n+1}\right)$. This shows that the proposed method (PR-ADI methods) in Eq. (7) is stable.

Definition 1: For linear difference operator $D_{h k}($.$) is$ stable if, for sufficiently small mesh size $h$ and $k$, there is a constant " $G$ " in dependent of size $h$ and $k$ such that

$$
\left|\varphi_{j n}\right| \leq G\left\{\max \left(\left|\varphi_{00}\right|,\left|\varphi_{M N}\right|\right)+\max _{1 \leq j \leq M-1}\left|D_{h k} \varphi_{j n}\right|\right\}
$$

Where $\varphi_{j n}$ is any mesh function. By adding the first and second half of Eq.(7) we obtain the differential equation given as:

$$
\begin{gathered}
u_{j n-1}^{p+1}-2 \beta u_{j n}^{p+1}-u_{j n+1}^{p+1} \\
+2\left[(\beta-\alpha) u_{j+1 n}^{p+1 / 2}-\beta u_{j n}^{p+1 / 2}+(\beta+\alpha) u_{j-1 n}^{p+1 / 2}\right] \\
=u_{j n+1}^{p}-u_{j n-1}^{p} \\
\mathrm{E} u_{j n}^{p+1}+2 F u_{j+1 n}^{p+1 / 2}=H u_{j n+1}^{p}
\end{gathered}
$$

$$
\begin{gathered}
E=\operatorname{dig}(N, 0,-2 \beta)+\operatorname{diag}(N,-1,-1)+\operatorname{diag}(N, 1,1), \\
F=\operatorname{diag}(N, 0,-\beta)+\operatorname{diag}(N,-1,(\beta+\alpha)) \\
+\operatorname{diag}(N, 1,(\beta-\alpha))
\end{gathered}
$$

and

$H=\operatorname{diag}(N, 0,0)+\operatorname{diag}(N,-1,1)+\operatorname{diag}(N,-1,-1)$.

Theorem 1: The difference operator defined in Eq.(18) is stable any for constant $\mathrm{G}$.

Proof: Let $D_{h k}$ and $L_{h k}$ are denoted the difference operator that defined on the left and right-hand side of Eq.(18) respectively and $\varphi_{j n}$ is any mesh function satisfies

$$
D_{h k}\left(\varphi_{j n}\right)=L_{h k}\left(\varphi_{j n}\right)
$$

where $L_{h k}(u)=H u_{j n+1}^{p}$. If the max $\left|\varphi_{j n}\right|$ occur at $(j n)=$ $(0,0)$ or $(j n)=(M, N)$ from definition 1 , the difference equation is stable. Let us consider $\max \left|\varphi_{j n}\right|$ exist at $(j n)=$ $(0,0)$. from Eq.(19) we have:

$$
\begin{gathered}
\left\|L_{h k}\left(\varphi_{00}\right)\right\|=\left\|D_{h k}\left(\varphi_{00}\right)\right\| \Rightarrow\left\|H u_{01}^{p}\right\| \\
=\left\|\mathrm{E} u_{00}^{p+1}+2 F u_{10}^{p+1 / 2}\right\| \\
\Rightarrow\left\|H \varphi_{01}^{p}\right\| \leq\left\|\mathrm{E} \varphi_{00}^{p+1}\right\|+2\left\|F \varphi_{10}^{p+1 / 2}\right\| \\
\left\|\varphi_{01}^{p}\right\| \leq\|\mathrm{E}\|\left\|\varphi_{00}^{p+1}\right\|+2\|F\|\left\|\varphi_{10}^{p+1 / 2}\right\| \\
\left\|\varphi_{01}^{p}\right\| \leq G\left\{\|\mathrm{E}\|\left\|\varphi_{00}^{p+1}\right\|+2\|F\|\left\|\varphi_{10}^{p+1 / 2}\right\|\right\}
\end{gathered}
$$

where $G=\frac{1}{\|H\|}$ is constant. Hence the difference equation is stable. Therefore this condition is true for $(j, n)=(M, N)$. Now for any interior point $(j, n)=(2(1) M-1,2(1) N-$ 1) we have:

$$
\begin{aligned}
& \left\|L_{h k}\left(\varphi_{j n}\right)\right\|=\left\|D_{h k}\left(\varphi_{j j}\right)\right\| \Rightarrow\left\|H u_{j n+1}^{p}\right\| \\
& =\left\|\mathrm{E} u_{j n}^{p+1}+2 F u_{j+1 n}^{p+1 / 2}\right\| \\
& \Rightarrow\left\|H \varphi_{j n+1}^{p}\right\|=\left\|\mathrm{E} \varphi_{j n}^{p+1}+2 F \varphi_{j+1 n}^{p+1 / 2}\right\| \\
& || H|||| \varphi_{j n+1}^{p}|| \leq|| \mathrm{E}|||| \varphi_{j n}^{p+1}||+2|| F|||| \varphi_{j+1 n}^{p+1 / 2}|| \\
& \left\|\varphi_{j n+1}^{p}\right\| \leq G\left\{\frac{1}{2\|F\|}\left(\varphi_{00}^{p+p+1 / 2}, \varphi_{M N}^{p+p+1 / 2}\right)\right. \\
& \left.+\frac{1}{\|E\|} \max _{\substack{2 \leq j<M \\
2 \leq j<N}}\left\|D_{h k}\left(\varphi_{j n}^{p+1 / 2}\right)\right\|\right\}
\end{aligned}
$$

where $G=2\|E\|\|F\|_{\|H\|}$ provided that all matrix norms are different from zero. The in equality in Eq. (17) is held. So the difference equation in Eq. (18) is stable. This implies that the 
solution to the system of equation in Eq. (7) is uniformly bounded and independent of mesh-size $\mathrm{h}$ and $\mathrm{k}$. Therefore the scheme is stable.

Corollary 1. Under the condition of the above theorem, the error $\varepsilon_{j n}$ between the exact solution $u\left(x_{j}, t_{n}\right)$ and the numerical solution $u_{j n}$ givenas $\varepsilon_{j n}=u\left(x_{j}, t_{n}\right)-u_{j n}$ with boundary and initial condition satisfy:

$$
\left|\varepsilon_{j n}\right| \leq G \max _{\substack{2 \leq j \leq M \\ 2 \leq j \leq N}}\left|T_{j n}\right|
$$

where $T_{j n}$ localtruncationerrorinEq.(6).

Proof: Let $\varepsilon_{j n}$ be the error and under the given condition its satisfies

$$
D_{h k}\left(\varepsilon\left(x_{j}, t_{n}\right)\right)=D_{h k}\left(u\left(x_{j}, t_{n}\right)-u_{j n}\right)=T_{j n}
$$

where $\mathrm{j}=2$ (1) $\mathrm{M}-1, \mathrm{n}=2$ (1) $\mathrm{N}-1$ and $\varepsilon_{00}=\varepsilon_{M N}=0$. By the above theorem stability of $D_{h k}$ implies that

$$
\begin{gathered}
\left|u\left(x_{j}, t_{n}\right)-u_{j n}\right|=\left|\varepsilon_{j n}\right| \leq G \max _{\substack{2 \leq j \leq M \\
2 \leq j \leq N}}\left|T_{j n}\right| \\
=G \max _{\substack{2 \leq j \leq M-1 \\
2 \leq j \leq N-1}}\left|\left(\frac{a k h^{2}}{3} \partial_{x}^{3}+\frac{k^{3}}{3} \partial_{t}^{2}+\frac{d k h^{2}}{6} \partial_{x}^{4}\right) u_{j n}\right|
\end{gathered}
$$

Thus the error is bounded and the scheme is stable. From this as $(h, k) \rightarrow(0,0)$ the maximum local truncation is tending to zero. It means $(h, k) \rightarrow(0,0), \max _{\substack{1 \leq j \leq M \\ 0 \leq j \leq N}}\left|T_{j n}\right| \rightarrow$ 0 . Again the scheme is consistent. Therefore the proposed scheme is convergent.

\section{Criteria for Investigating the Accuracy of the Method}

In this section, we investigate the accuracy of the present method. There are two types of errors Round-off errors and Truncation errors occur when differential equations are solved numerically. Rounding errors originate from the fact that computers can only represent numbers using a fixed and limited number of significant figures. Thus, such numbers or cannot be represented exactly in computer memory. The discrepancy introduced by this limitation is called Round-off error. Truncation errors in numerical analysis arise when approximations are used to estimate some quantity. The accuracy of the solution is depend on how small we make the step size, h, and time step $\mathrm{k}$. To test the performance of the proposed method, $\mathrm{L}_{2}$ and $\mathrm{L}_{1}$ is used to measure the accuracy of the method. This error norm are calculated as followed in [14] is given by

$$
\begin{gathered}
L_{1}=\max _{1 \leq n \leq M}\left|u\left(x_{j}, t_{n}\right)-u_{j n}\right|, \\
L_{2}=\sqrt{\frac{1}{M} \sum_{j=0}^{M}\left|u\left(x_{j}, t_{n}\right)-u_{j n}\right|^{2}} \\
E_{\text {num }=\frac{1}{M} \sum_{j=0}^{M}\left|u\left(x_{j}, t_{n}\right)-u_{j n}\right|}
\end{gathered}
$$

where $M$ is the maximum number of step, $u\left(x_{j}, t_{n}\right)$ is the exact solution and $u_{j n}$ approximation solution of advectiondiffusion equation in Eq.(1) at the grid point $\left(x_{j}, t_{n}\right)$.

\section{Numerical Experiments}

To test the validity of the proposed method, we have considered the following three model problem. Numerical results and errors are computed and the outcomes are represented tabular and graphically.

Example1: Consider the advection-diffusion equation considered by Sigrunin [15] given as:

$u_{t}+a u_{x}=d u_{x x}+\mathrm{g}(\mathrm{x}, \mathrm{t}),-\pi \leq x \leq \pi, 0 \leq t \leq T$,

And the exact solution is

$$
u(x, t)=e^{-d t} \sin (x-a t)
$$

Example2: Consider the advection-diffusion equation considered by Abdullahand Khatunin [11] given as:

$u_{t}+a u_{x}=d u_{x x}, 0 \leq x \leq 50,1 \leq t \leq 60$

And the exact solution is

$$
u(x, t)=\frac{1}{\sqrt{4 \pi d t}} e^{(x-a t)^{2} / 4 d t}
$$

for $\mathrm{a}=0.3 \mathrm{~m} / \mathrm{s}, d=.005 \mathrm{~m} 2 / \mathrm{s}$.

Example3: Consider the advection-diffusion equation considered by Appadu in [1] given as:

$$
u_{t}+a u_{x}=d u_{x x}, 0 \leq x \leq 1,0 \leq t \leq 1
$$

and initial and boundary condition is given as

$$
\begin{gathered}
u(x, 0)=e^{-\left(\frac{x+0.5}{0.00125}\right)^{2}}, \\
u(0, t)=\frac{0.025}{\sqrt{0.000625+0.02 t}} e^{-\left(\frac{0.5-t}{0.00125+0.04 t}\right)^{2}}, \\
u(0, t)=\frac{0.025}{\sqrt{0.000625+0.02 t}} e^{-\left(\frac{1.5-t}{0.00125+0.04 t}\right)^{2}} .
\end{gathered}
$$

Table 1. Comparison of $L_{2}$ errors and experimental order of convergence (EOC) concerning the classical diffusion fluxes for the exponentially growing solution with $a=1$ and $d=0.1$ for example one. Computations carried out until final time $T=10$ with time step $h=k$.

\begin{tabular}{llllll}
\hline & No of grid & Sigrun in [15] (BR2) & & Present Method & \\
\hline $\mathbf{M}$ & $\mathbf{N}$ & $\boldsymbol{L}_{\mathbf{2}}$ & $\mathbf{E O C}$ & $\boldsymbol{L}_{\mathbf{2}}$ & EOC \\
\hline 20 & 20 & $1.03 \mathrm{E}-01$ & & $1.6288 \mathrm{E}-08$ & 2.2297 \\
40 & 40 & $2.36 \mathrm{E}-02$ & 2.13 & $6.2484 \mathrm{E}-08$ & 2.6962 \\
80 & 80 & $5.63 \mathrm{E}-03$ & 2.07 & $2.2977 \mathrm{E}-09$ & 3.1788 \\
160 & 160 & $1.38 \mathrm{E}-03$ & 2.02 & $8.2810 \mathrm{E}-10$ & 3.6699 \\
320 & 320 & $3.44 \mathrm{E}-04$ & 2.01 & $8.2671 \mathrm{E}-10$ & 3.9218 \\
640 & 640 & $8.60 \mathrm{E}-05$ & 2.00 & $3.1289 \mathrm{E}-11$ & 4.1193 \\
\hline
\end{tabular}


Table 2. Expiration of $E_{\text {num }}$-errors, $L_{1}$-errors $L_{2}$-errors and experimental order of convergence (EOC) for the classical diffusion fluxes for the exponentially growing solution of [15] Example 2 with $a=0.03$ and $d=0.005$.

\begin{tabular}{lllll}
\hline Step size & \multicolumn{5}{l}{ Present methods } \\
\hline $\mathbf{h}$ & $\mathbf{k}$ & $\boldsymbol{E}_{\text {num }}$ & $\boldsymbol{L}_{\mathbf{1}}$ & $\boldsymbol{L}_{\mathbf{2}}$ \\
\hline 2.5 & 3 & $2.4273 \mathrm{E}-07$ & $5.3400 \mathrm{E}-6$ & $1.1385 \mathrm{E}-06$ \\
1.25 & 1.5 & $1.2794 \mathrm{E}-06$ & $5.3733 \mathrm{E}-05$ & $8.2912 \mathrm{~T}-06$ \\
0.83 & 1 & $4.6734 \mathrm{E}-06$ & $2.8975 \mathrm{E}-04$ & $3.6799 \mathrm{E}-05$ \\
0.625 & 0.75 & $8.6415 \mathrm{E}-06$ & $7.0860 \mathrm{E}-04$ & 2.6962 \\
0.5 & 0.6 & $1.2005 \mathrm{E}-06$ & $1.2245 \mathrm{E}-04$ & 2.9771 \\
\hline
\end{tabular}

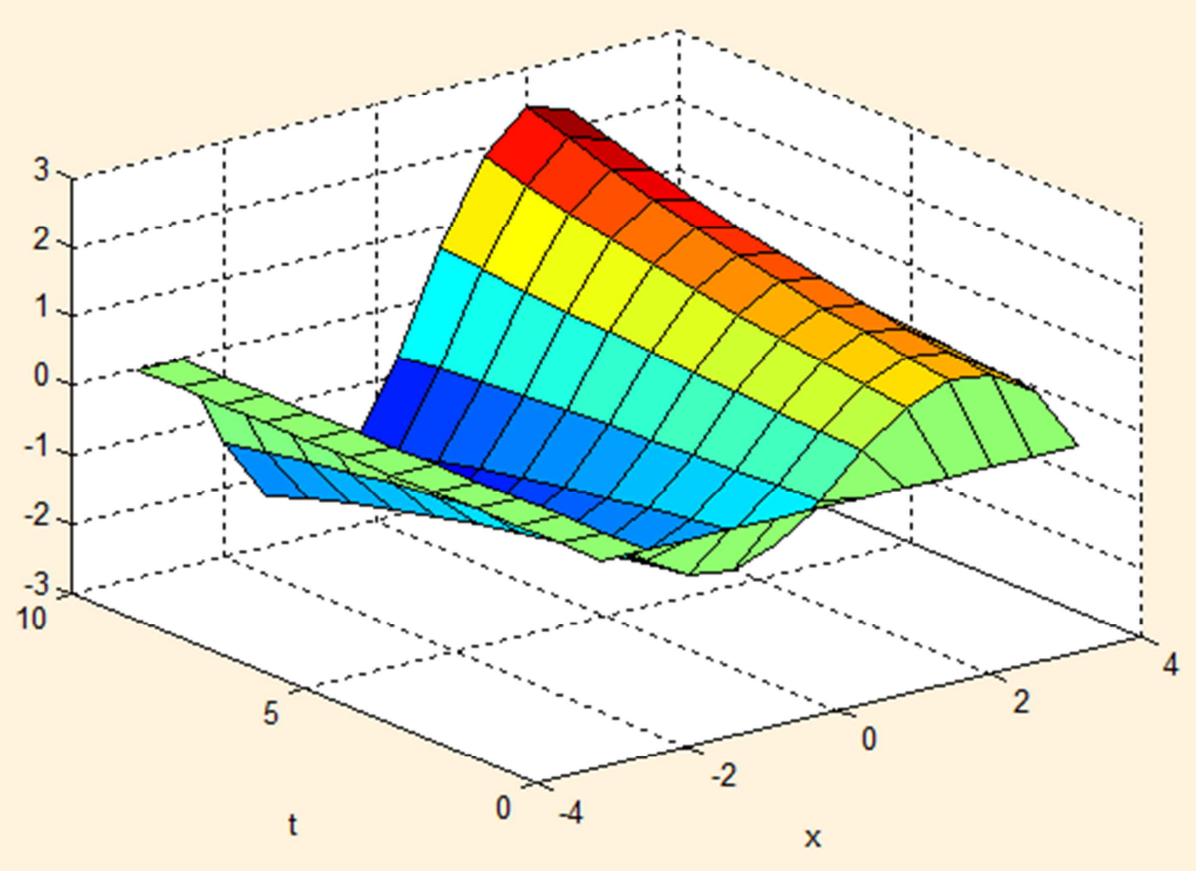

a) The surface of numerical solution of example one $N=M=80$.

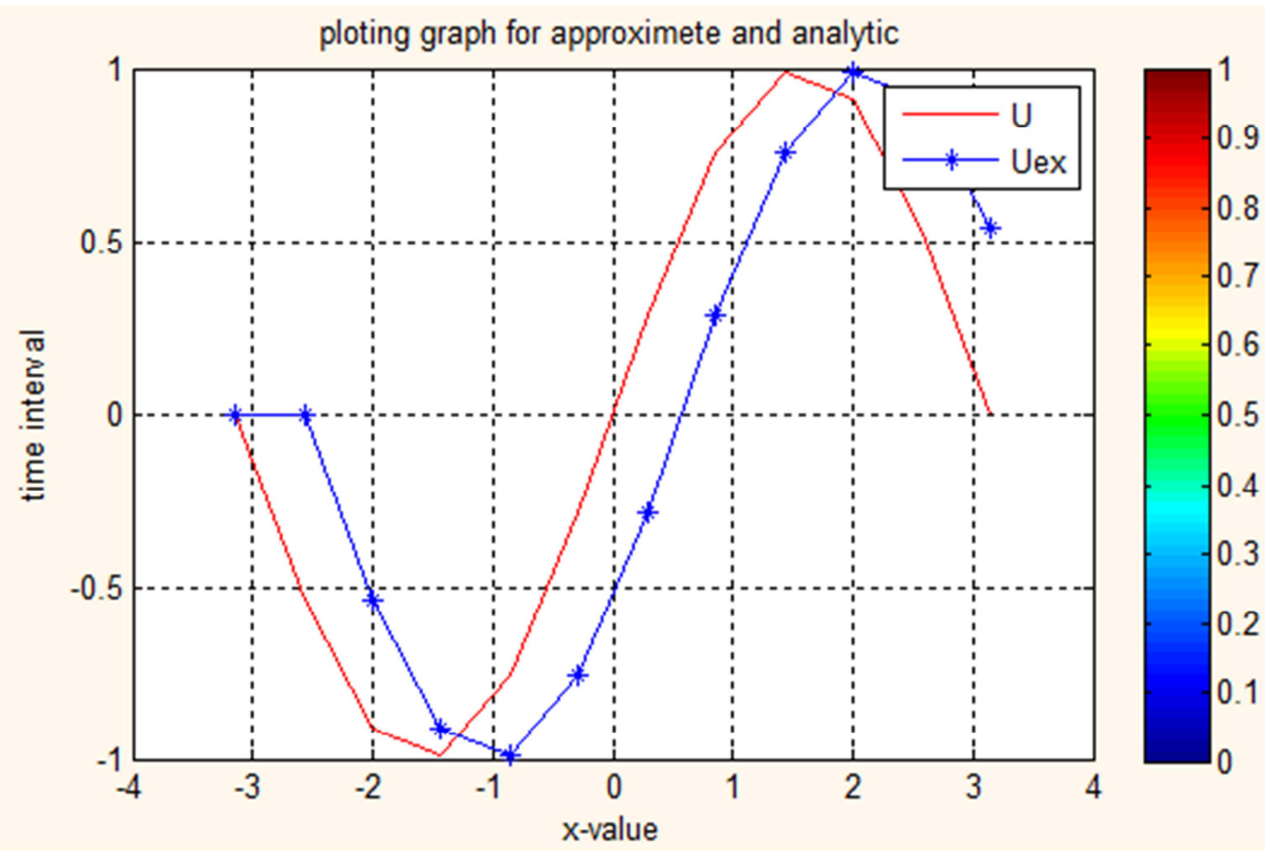

b) plotting graphs of numerical versus exact solutions $\mathrm{M}=9$.

Figure 1. Graphs of numerical and exact solution of example one for $h=k=0.5$. 

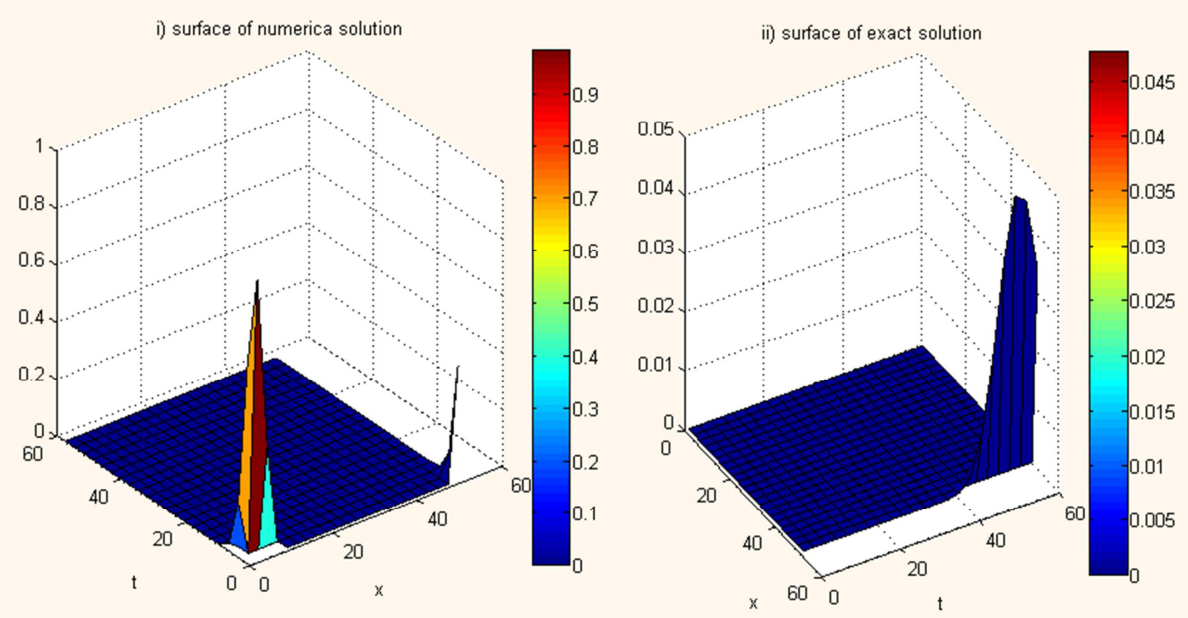

Figure 2. Graphs of numerical solution of example two for $k=3, h=2.5$.

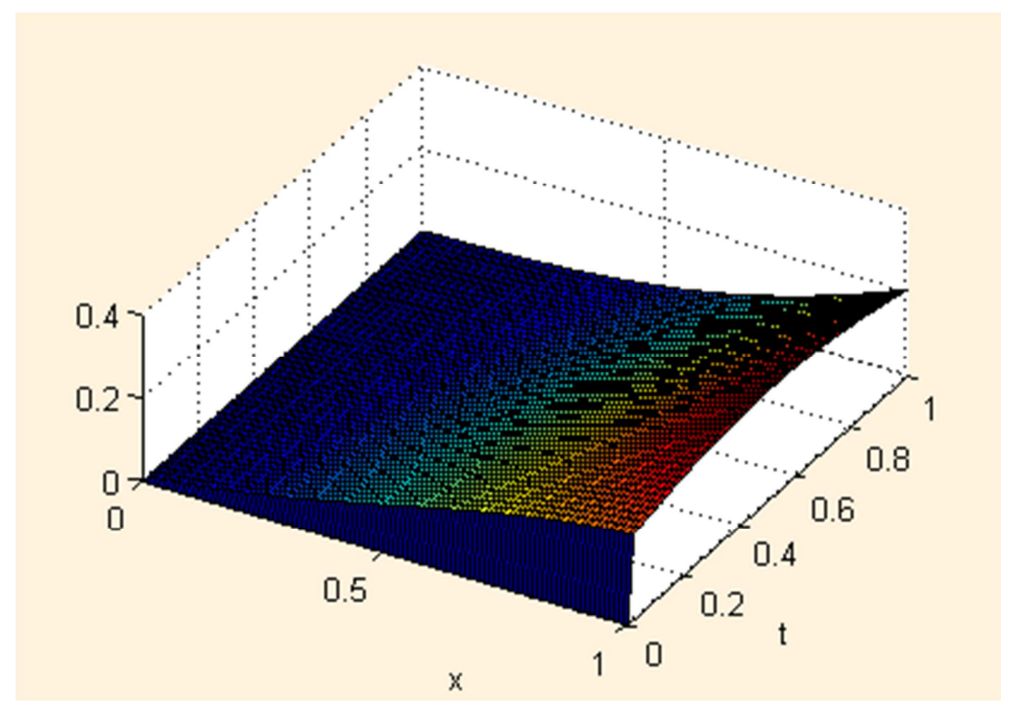

a) The surface of numerical solution for $h=0.05, k=0.1$.

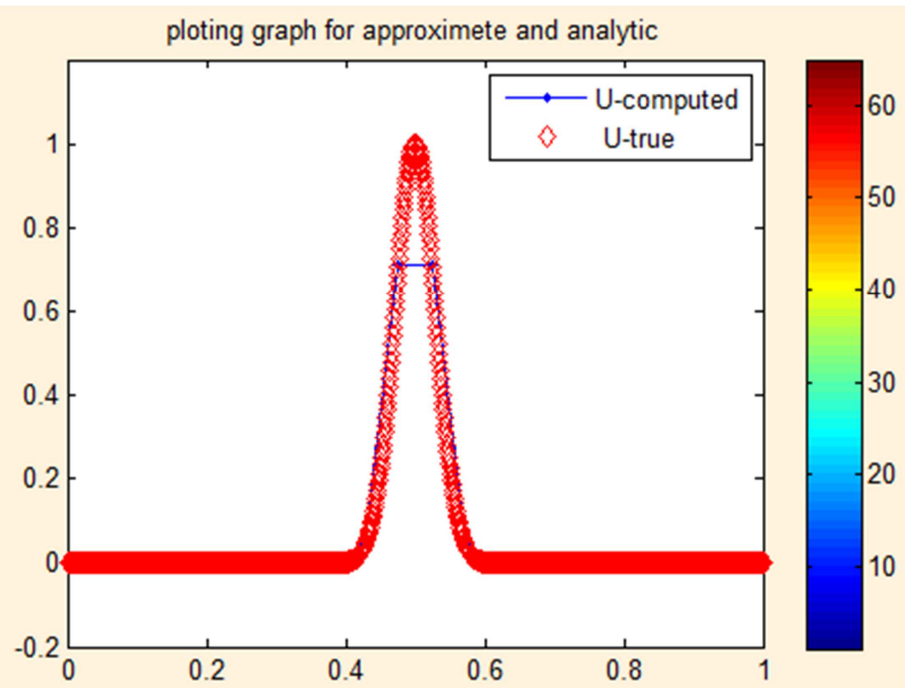

b) Plotting graphs of numerical versus exact solutionk $=0.01, \mathrm{~h}=0.02$.

Figure 3. Graphs of numerical and exact solution for example three. 
Table 3. Computations of $E_{\text {num }}$-errors, $L_{\infty}$-errors $L_{2}$-errors and experimental order of convergence (EOC) for the classical diffusion fluxes for the exponentially growing solution [15] of Example 3 with $a=1$ and $d=0.1$.

\begin{tabular}{|c|c|c|c|c|c|}
\hline \multicolumn{2}{|l|}{ Mesh size } & \multicolumn{4}{|c|}{ By present method } \\
\hline h & $\mathbf{k}$ & $E_{\text {num }}$ & $\boldsymbol{L}_{\infty}$ & $L_{2}$ & EOC \\
\hline 0.005 & 0.02 & $2.3352 \mathrm{E}-07$ & $4.7350 \mathrm{E}-05$ & $3.3189 \mathrm{E} 06$ & 3.8291 \\
\hline 0.01 & 0.02 & $4.6246 \mathrm{E}-07$ & $4.7170 \mathrm{E}-05$ & $4.6706 \mathrm{E}-06$ & 3.3362 \\
\hline 0.02 & 0.04 & $9.0713 \mathrm{e}-07$ & $4.7171 \mathrm{e}-05$ & $6.5414 \mathrm{e}-06$ & 2.8502 \\
\hline \multicolumn{6}{|c|}{ Appadu in [1] } \\
\hline 0.005 & 0.02 & $1.8166 \mathrm{E}-04$ & $5.8157 \mathrm{E}-04$ & $1.6348 \mathrm{E}-04$ & 0.25 \\
\hline 0.01 & 0.02 & 7.3296E-04 & 0.0024 & $1.6348 \mathrm{E}-04$ & 0.50 \\
\hline 0.02 & 0.04 & $1.2252 \mathrm{E}-04$ & $3.7946 \mathrm{E}-04$ & $3.7946 \mathrm{E}-04$ & 0.50 \\
\hline
\end{tabular}

\section{Discussion and Conclusion}

In this paper, Peaceman-Rachford alternating direct implicit method is applied and presented for solving linear advection-diffusion equation. To demonstrate the competence of the method, it is applied on three model examples by taking different values for mesh size, $\mathrm{h}$, and $\mathrm{k}$. Numerical results obtained by the present method has been computed with numerical results obtained by the methods in $[1,11,15]$ and they are summarized and presented in Tables and graph. In this paper both theoretical and numerical error bounds has been established for the Peaceman-Rachford alternating direct implicit method. As can be seen from the numerical results in terms of maximum absolute error, Numerical Error with order of convergence that predicted in tables and graphs above, the present method is superior to the method developed in [1, $11,15]$ and approximates the exact solution very well. Further, as shown in Figures1-3, the proposed method approximates the exact solution very well for which most of the current methods fail to give good results. To further verify the applicability of the planned method, graphs were plotted aimed at the above examples for exact solutions versus the numerical solutions obtained with their errors. As Figure 1 (b) indicates numerical solution obtained by present method is good a agreement of the results with exact solutions, which proves the reliability of the method for example1. Figures 2 indicate the numerical solution has a good agreement of the results with the exact solution for example 2. Figures 3 (b) indicate the numerical solution has a good agreement of the results with the exact solution for example 3. Further, the numerical results presented in this paper validate the improvement of the proposed method over some of the existing methods described in the literature.

\section{Availability of Data and Materials}

All data generated or analyzed during this study are included.

\section{Competing Interests}

The authors declare that they have no competing interests.

\section{Authors' Contributions}

'KA' proposed the main idea of this paper. He prepared the manuscript and performed all the steps of the proofs in this research paper. The author read and approved the final manuscript.

\section{Acknowledgements}

The author wish to express his thanks to the authors of literatures for the provision of initial idea for this work.

Last but not least, the author wish to express his thanks to all his beloved friends; your kindness and helps will be a great memory for me.

\section{References}

[1] Appadu, Appanah Rao. Numerical solution of the 1D advection-diffusion equation using standard and non-standard finite differences scheme. Journal of Applied Mathematics, 2013.

[2] Jiaqi Zhong, Cheng Zeng, Yupeng Yuan, Yuzhe Zhang, Ye Zhang. Numerical solution of the unsteady diffusionconvection-reaction equation based on improved spectral Galerkin method. AIP Advances, 8 (4), 2018.

[3] Evstigneev, M. Nikolay. Numerical analysis of Krylov multi-grid methods for stationary advection-diffusion equation. International Journal of Physics: Conference Series, 1391 (1), 2019.

[4] Soyoon Bak, Philsu Kim, Xiangfan Piao, Sunyoung Bu. Numerical solution of advection-diffusion type equation by modified error corrections scheme. Open Springer journal of Advances in Difference Equations, 2018.

[5] Yu Liu, Marcial Gonzalez, Carl Wassgren. Modeling Granular Material Blending in a Rotating Drum Using a Finite Element Method and Advection-Diffusion Equation Multi-scale Model. American Institute of Chemical Engineers, 64 (9), 3277-3292, 2018.

[6] AVCI Derya, Aylin Yetim. Analytical solutions to the advection-diffusion equation with the Atangana-Baleanu derivative over a finite domain", Ballkesir Universities Fen Balmier Enstitüsü Dergisi, 20 (2) 382-395, 2018.

[7] Abdelkader Mojtabi, Michel Deville. One-dimensional linear advection-diffusion equation: Analytical and finite element solution. Computers and Fluids, Elsevier, 107, 189-195, 2015. 
[8] Cotta RM. Integral transforms in computational heat and fluid flow. Boca Raton (FL): CRC Press; 1993.

[9] V. A. N. Genuchten, Martinus TH, Feike J. Leij, Todd H. Skaggs, Nobuo Toride, Scott A. Bradford, Elizabeth M. Pontedeiro. Exact analytical solutions for contaminant transport in rivers 1 . The equilibrium advection-dispersion equation. Journal of Hydrology and Hydromechanics, 61 (2), 146-160, 2013.

[10] Guerrero, J. S. Pérez, Luiz Cláudio Gomes Pimentel, Todd H. Skaggs, and M. T. H. Van Genuchten. Analytical solution of the advection-diffusion transport equation using a change-ofvariable and integral transform technique. International Journal of Heat and Mass Transfer 52 (13) 3297-3304, 2009.

[11] L.S. Andallah, M. R. Khatun. Numerical solution of advection-diffusion equation using finite difference schemes. Bangladesh Journal of Scientific and Industrial Research, 55 (1) $15-22,2020$.

[12] W. H. Hundsdorfer, J. G. Verwer. Stability and Convergence of the Peaceman-Rachford alternative direct implicitly method for Initial-Boundary Value Problems. Journal of mathematics of computation, 53 (187), 81-101, 1987.

[13] Argourlay. Splitting methods for time-dependent partial differential equations. The State of the Art in Numerical Analysis s (D. Jacobs, ed.), Academic Press, New York, pp. 757-791, 1977.

[14] Gurhan Gurarslan, Halil Karahan, Devrim Alkaya, Murat Sari, Mutlu Yasar. Numerical Solution of Advection-Diffusion Equation Using a Sixth-Order Compact Finite Difference Method. Hindawi Publishing Corporation Mathematical Problems in Engineering. 2013 (7), 2013.
[15] Sigrun Ortleb. $\mathrm{L}^{2}$-stability analysis of IMEX- $(\sigma, \mu)$ D schemes for linear advection-diffusion equations. Applied Numerical Mathematics, 147, 43-65, 2020.

[16] D. M. William, A. Jameson. Energy stable Flux Reconstruction Schemes for Advection-Diffusion Problems on Tetrahedral. Journal of Scientific Computing, 2013.

[17] Millard H. Alexander, Jane E. Smedley, Gregory C. Corey. On the physical origin of propensity rules in collisions involving molecules in $\Sigma$ electronic states, The Journal of Chemical Physics, 1986.

[18] Philsu Kim, Soyoon Bak. Algorithm for a cost reducing timeintegration scheme for solving incompressible Navier-Stokes equations. Computer Methods in Applied Mechanics and Engineering, 2021.

\section{Biography}

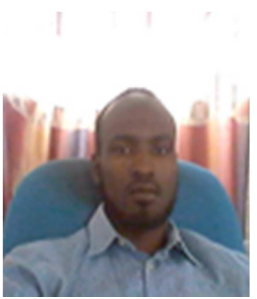

Kedir Aliyi Koroche is Graduate B. sc in Mathematics from Ambo University in 2016G. C. and M. Sc. in Mathematics (specialized in Numerical Analysis) from Jimma University in 2019G. C. Now he works at Ambo University as Lecturer and Researcher. He conducts research on Numerical Analysis. His topics of research in numerical Analysis include: Numerical solutions of ODEs, PDEs using single step and Multistep methods, Finite difference, Differential Quadrature methods. 